\title{
A STUDY OF BREAST CANCER IN KORLE BU TEACHING HOS- PITAL: ASSESSING THE IMPACT OF HEALTH EDUCATION
}

\author{
*J.N.A. CLEGG-LAMPTEY AND W.M. HODASI \\ Department of Surgery, University of Ghana Medical School, P.O. Box 4236, Accra.
}

\section{SUMMARY}

Introduction: Many patients with breast cancer report late with advanced disease. It is not known if recent breast awareness education programmes have led to a change in this trend at the Korle $\mathrm{Bu}$ Teaching hospital (KBTH).

Method: A prospective study of the characteristics of breast cancer patients seen by a surgical unit at KBTH over a three year period.

Results: There were 158 patients, 156 females and 2 males. The age group most commonly affected was 40-49. The upper outer quadrant of the breast was affected in $67 / 158(42.4 \%)$ and all quadrants affected in $29 / 158(18.4 \%)$. Ninety one (57.6\%) had Stage III - IV disease and the average duration of symptoms was 10 months. Average tumour size was $6 \mathrm{~cm} \times 7 \mathrm{~cm}$. Diagnosis was by triple assessment with Fine needle aspiration cytology the most frequently used pathological investigation. Invasive ductal carcinoma was the commonest pathological type (115/134). Eighty three (52.5\%) had mastectomy and $12(7.6 \%)$ had wide local excision. Neoadjuvant chemotherapy was given to $77 / 123(62.6 \%), 5$ of whom had complete pathological response. Fifty five $(34.8 \%)$ were lost to follow up: 20 before treatment commenced, 15 during or after neoadjuvant chemotherapy and 14 after treatment. Eleven developed lymphoedema. There were 42 metastatic events affecting 35 patients during follow-up, including pleura (11), brain (10) and lungs (9).

Conclusions: Breast cancer continues to affect a young population and patients still present late with advanced disease. Education needs to be intensified, but research into the reasons for late presentation will help address the reasons/misconceptions responsible for this state of affairs.

Keywords: Breast cancer, Korle $\mathrm{Bu}$, pathology, diagnosis, treatment

\footnotetext{
* Author for correspondence
} clegglamptev@hotmail.com

\section{INTRODUCTION}

Breast cancer is the leading malignancy in Ghana ${ }^{1}$. It accounts for $15.4 \%$ of all malignancies and appears to be on the increase ${ }^{1}$. In $199612.8 \%$ of all admissions for malignant neoplasms to the Korle $\mathrm{Bu}$ Teaching hospital were for breast cancer ${ }^{2}$. There have been many public education programmes about breast cancer within the last few years and some non-governmental organisations like Mammocare Ghana, Reach for Recovery and the Cancer Society of Ghana have been formed to create breast cancer awareness in the general public.

Fifty percent or more of Ghanaians with breast cancer report to hospital with advanced disease ${ }^{3,4}$. On average they report 8 months or more after first noticing a change in their breasts ${ }^{4}$. Many of these patients are referred to the Korle $\mathrm{Bu}$ Teaching hospital where they are seen at the surgical outpatients clinic. It is not known whether public education on breast cancer has had an impact on the characteristics of breast cancer cases reporting to the surgical units of the Korle $\mathrm{Bu}$ Teaching hospital. We prospectively studied the case histories of patients referred to one unit at Korle $\mathrm{Bu}$ over a three-year period to determine any changes in breast cancer presentation.

\section{METHODS}

Patients with carcinoma of the breast referred to one of the four surgical units at the Korle $\mathrm{Bu}$ Teaching hospital were studied prospectively over a three-year period to determine the ages, presenting complaint, duration of symptoms, stage at presentation and the site of the tumour. The treatment given - type of surgery, chemotherapy and referral to radiotherapy - was also studied.

Patients were first seen at the Out-patients clinic where a detailed history was taken and fine needle aspiration cytology (FNAC) performed on all breast lumps, except for very obvious fibroadenomas in young women under 20. Core biopsies 
were performed on the ward if FNAC was not possible or if FNAC results were equivocal. The indications for incision biopsy were ulcerated tumours/skin nodules, Paget's disease of the nipple and inflammatory breast cancer, where no mass was palpable. Excision biopsies were performed in theatre under local anaesthetic. Mammograms and/or ultrasound were requested especially if there was no palpable lump (e.g. some cases of inflammatory breast cancer) or if breast conservation surgery was contemplated. Investigations to determine metastases were carried out once a diagnosis of breast cancer was made. These included a chest X-ray, liver function tests, ultrasound scan of the liver and bone scan. X-ray of symptomatic bones was requested when bone scans were not available. Each patient was staged by the American Joint Committee on Cancer (AJCC) TNM staging $^{5}$. A treatment plan was then drawn for each patient. Where further discussion was required the patient was referred to the Korle Bu Breast Clinic for discussion by a multidisciplinary team.

Patients requiring surgery were booked for surgery while those requiring neoadjuvant chemotherapy (those with locally advanced tumours) were scheduled to have it on the ward on specific days. Surgery was scheduled for the latter patients after two-to-four cycles of chemotherapy, depending on tumour response. Patients with early-stage tumours had surgery followed by adjuvant chemotherapy. Patients with inoperable tumours were not offered surgery.

\section{RESULTS}

There were 158 patients seen, comprising 156 women and 2 men. The ages ranged from 24 to 75 years (Mean 48.1years, Median 47 years) (Figure $1)$.

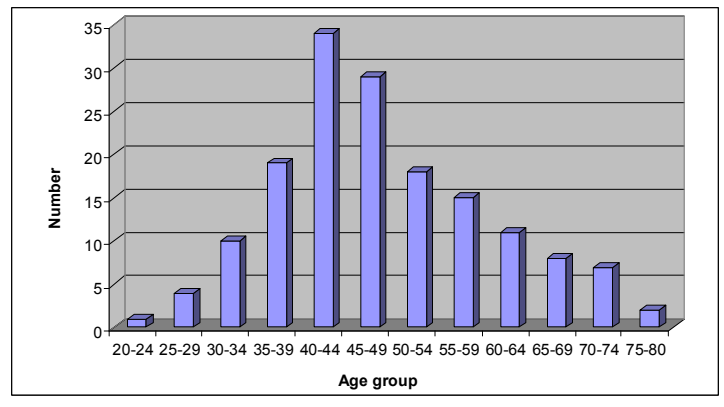

Figure 1 Age distribution of breast cancer patients

Sites of tumour: The cancer was on the left side in 76 cases and right in 82 . The sites involved are shown in Table 1.
Table 1 Sites of tumour

\begin{tabular}{lcc}
\multicolumn{1}{c}{ Site } & Number & Percentage \\
\hline Upper outer quadrant* & 67 & 42.4 \\
Central & 31 & 19.6 \\
Total (All quadrants)** & 29 & 18.4 \\
Lower outer quadrant & 15 & 9.5 \\
Upper inner quadrant & 9 & 5.7 \\
Lower inner quadrant & 5 & 3.2 \\
Axillary nodes*** & 2 & 1.3 \\
\hline Total & $\mathbf{1 5 8}$ & $\mathbf{1 0 0}$ \\
\hline
\end{tabular}

* Includes axillary tail

** Includes inflammatory cancer with no obvious mass

*** No breast mass

Stage of presentation: The stage of presentation by AJCC TNM is shown in Table 2.

Table 2 TNM stage of tumours

\begin{tabular}{ccc}
\hline & $\begin{array}{c}\text { T, N or M sta- } \\
\text { tus }\end{array}$ & $\begin{array}{c}\text { Number of pa- } \\
\text { tients }\end{array}$ \\
\hline T & Tx* & 3 \\
& Tis & 1 \\
T0 & 2 \\
T1 & 8 \\
T2 & 43 \\
T3 & 30 \\
T4a & 1 \\
& T4b & 56 \\
T4c & 2 \\
T4d & 12 \\
& N0 & 55 \\
& N1 & 71 \\
& N2 & 22 \\
& N3 & 10 \\
& Mx & 151 \\
& M1 & 7 \\
\hline Tumour size could not be determined in 3 previously excised tu-
\end{tabular}
mours

Duration of symptoms: The interval between when symptoms were first noticed to time of presentation ranged from 2 weeks to 5 years. The mean duration was 10 months and the median was 7 months.

Tumour size: The mean tumour size was $6 \times 7 \mathrm{~cm}$.

Method of pathological diagnosis: Overall 96 patients were diagnosed by FNAC, 50 by excision biopsy, 6 by core biopsy and 6 by incision biopsy. Of the 50 excision biopsies, 26 were excised before being referred to KBTH. Of the remaining 24, 14 had FNAC which were reported as either inconclusive or benign. Incision biopsies were used in the diagnosis of 3 ulcerated tumours, 2 inflammatory breast cancers and 1 Paget's disease of the 
nipple. FNAC was successful in diagnosing $87.3 \%$ of the cancers.

Pathology: A full histological diagnosis (following core biopsy or mastectomy/wide local excision specimen) was possible in 134 cases. The others were diagnosed as having malignant cells by FNAC but did not have tissue diagnosis because they did not have surgery. The tumour types are shown in Table 3 . Twenty four of the 55 patients with clinically negative nodes (cN0) had cancer on pathology (pN1). Conversely 16 patients with $\mathrm{cN} 1$ nodes had no cancer on pathology (pN0). All patients with $\mathrm{N} 2+$ nodes clinically did have cancer on pathology.

Table 3 Pathological type of tumours

\begin{tabular}{lcc}
\multicolumn{1}{c}{ Pathological type } & Number & Percentage \\
\hline Invasive ductal & 115 & 85.8 \\
Invasive lobular & 7 & 5.2 \\
Ductal Carcinoma In Situ & 3 & 2.2 \\
Medullary & 3 & 2.2 \\
Mucinous & 2 & 1.5 \\
Adenosqamous & 2 & 1.5 \\
Adenocarcinoma & 1 & 0.7 \\
Apocrine carcinoma & 1 & 0.7 \\
\hline Total & $\mathbf{1 3 4}$ & $\mathbf{9 9 . 8}$ \\
\hline
\end{tabular}

\section{Treatment:}

a. Surgery: Eighty three patients $(52.5 \%)$ had mastectomy and $12(7.6 \%)$ had wide local excision. Twenty two $(13.9 \%)$ were not suitable for breast surgery due to advanced disease and $41(25.9 \%)$ either absconded or refused surgery. Nine of the mastectomy specimen showed no residual tumour. Five of these followed neoadjuvant chemotherapy and 4 were mastectomies following pathologically incompletely excised tumours.

Two patients had latissimus dorsi myocutaneous flap breast reconstruction at the time of mastectomy. Both operations were done to cover a large defect in the skin following removal of the breast.

Level II axillary clearance was the routine method of axillary dissection employed in both mastectomy and wide local excision operations. b. Chemotherapy: Of the 158 patients seen, 123 needed chemotherapy. Seventy seven of them (62.6\%) had neoadjuvant and 46 (37.4\%) had adjuvant chemotherapy. The commonest cyclical combination therapy regime used was CAF (Cyclophosphamide, Adriamycin [doxorubicin] and 5-Fluorouracil) in 78 patients $(64.2 \%)$. The number of cycles given ranged from 1 (defaulters) to 6 . The mean number of cycles given was 4 .

c. Radiotherapy: Forty one patients were referred for post-operative radiotherapy on account of the advanced stage of their tumours on presentation. Other criteria for referral are listed in Table 4.

Table 4 Reasons for referral to radiotherapy

\begin{tabular}{lc}
\hline \multicolumn{1}{c}{ Indication } & $\begin{array}{c}\text { Number } \\
\text { Referred }\end{array}$ \\
\hline Following wide local excision & 12 \\
T4 Tumours, post-mastectomy & 25 \\
Incompletely excised tumour. E.g. & 14 \\
muscle involvement & \\
$\geq 4$ lymph nodes involved with tu- & 9 \\
mour & 15 \\
Metastatic disease (Brain, bones) & \\
\hline Note: Some of the groups overlap
\end{tabular}

Note: Some of the groups overlap

Defaulting patients: Fifty five patients (34.8\%) were lost to follow-up during the period of the study. Of these $14(8.9 \%)$ completed their treatment. Twenty patients (12.7\%) defaulted before treatment; $6(3.8 \%)$ defaulted and returned after many months with Stage 4 disease; 15 (9.5\%) discontinued treatment during or after neoadjuvant chemotherapy.

Table 5 Sites of metastases during 3 year period

\begin{tabular}{lc}
\hline \multicolumn{1}{c}{ Site } & Number \\
\hline Pleura (effusion) & 11 \\
Brain & 10 \\
Pulmonary & 9 \\
Liver & 6 \\
Bones & 5 \\
Ovarian & 1
\end{tabular}

Complications: Eleven patients developed lymphoedema following surgery. There were 42 metastatic events affecting 35 patients during the follow-up period (Table 5). One patient had diathermy burns on her thigh during surgery due to faulty 
diathermy equipment. Nausea and vomiting was present to a degree in all patients who received chemotherapy. All patients receiving Adriamycin developed alopecia. Seromas that needed aspiration occurred in 23/95 patients following axillary node dissection. Fourteen others had mild seromas that settled spontaneously.

\section{DISCUSSION}

Cancer of the breast affects a relatively young population in Ghana. The majority of our patients were in the fifth decade (40-49), similar to previous studies $^{4,6}$ and this confirms the occurrence of breast cancer in Ghana about a decade earlier than in Caucasians ${ }^{1,4}$.

Male breast cancer remains relatively uncommon. Although most texts put the male incidence at $1 \%$ of breast cancers ${ }^{7}$ the proportion in various countries is variable. Analysis of pathology specimen in Ghana puts the incidence at $2 \%{ }^{8}$ and in Jos (Nigeria) it has been reported as high as $8.6 \%$. In this series it is $1.3 \%$.

The upper outer quadrant was the commonest site involved in breast cancer. The number of patients with cancer involving the whole breast, 29/158 $(18.4 \%)$ is a reflection of how advanced most of the tumours were. Some of these patients had inflammatory breast cancer with no definite palpable lump.

\section{Stage of disease}

As Table 2 shows, not many patients presented with early disease. Ninety one patients $(57.6 \%)$ had advanced disease at presentation. Seventy patients $(45.2 \%)$ had either peau d'orange or ulcerated tumours. The tumours were large, $6-7 \mathrm{~cm}$ on average. Earlier studies in Ghana have shown similar proportion of breast cancer patients presenting with advanced disease $\mathrm{e}^{3,4}$. It thus appears that public education has so far failed to change this unfortunate trend.

\section{Duration of symptoms}

Although we did not find out the reasons for late reporting, the mean duration of symptoms of 10 months is worrying. It is slightly longer than was found from the same hospital five years earlier ${ }^{4}$, indicating no impact of breast awareness education programmes. Some of the patients reporting to us after two years had had previous hospital treatment and defaulted, presumably to try alternative treatment.

\section{Method of diagnosis}

Pathological diagnosis was mainly by fine needle aspiration cytology. Unfortunately some of the patients referred to the unit had already had excision biopsy, many of them incompletely excised. Inadequately excised cancers often posed a problem. Sometimes the pathologist gave no information about resection margins. Where the tumour had not been completely excised the patient needed further surgery, often mastectomy. Some of these patients could have been spared a mastectomy if the initial biopsy had not been by simple excision.

It is recommended that breast lumps be properly investigated by triple assessment ${ }^{10}$. The sensitivity of FNAC was only $87.3 \%$, and therefore clinicians should always consider clinical evaluation and imaging (when necessary) before making or excluding the diagnosis of breast cancer. As shown by this study other forms of pathological diagnosis (core biopsy or incision biopsy) may sometimes be preferable. The resort to excision biopsies of all breast lumps including clinically malignant ones should be discouraged.

Incision biopsies were done in 6 patients. These patients had ulcerated tumours (3), inflammatory breast cancer (2) and Pagets disease (1). The two patients with inflammatory cancer had no palpable breast lumps. Their FNACs were negative as were core biopsies; the diagnosis was only confirmed by incision biopsy of the skin of the breast. Incision biopsies for all breast lumps should, however, be discouraged. The wounds might not heal, as happened in the case of two patients who presented with $\mathrm{T} 4 \mathrm{~b}$ disease (skin involvement) which they did not have before incision biopsy.

\section{Treatment}

Mastectomies constituted most of the operations for breast cancer. Wide local excision (breast conservation) was done in only 12 out of the 95 patients who had surgery. Breast conservation surgery therefore accounted for $12.6 \%$ (12/95) of our surgical procedures compared to up to $70-85 \%$ in the western world ${ }^{\mathbf{1 1}}$. This reflects the advanced state of tumours at presentation.

The criteria followed for breast conservation were $^{1,12}$ :

- Lumps not greater than $4 \mathrm{~cm}$.

- Lumps not centrally placed (i.e. not behind nipple/areola)

- No other tumour focus demonstrated in the breast (by mammogram or ultrasound) 
- Tumour not poorly differentiated

- Patient willing and able to have radiotherapy to the breast after surgery

- Breast not too small (Favourable breast: tumour ratio)

- Patient wanted breast conservation (provided other conditions were met). Some patients preferred mastectomy!

Patients treated by wide local excision always had level II axillary clearance as part of the surgical procedure and referred for post-operative radiotherapy to the remaining breast. Where tumours had already been completely excised before referral to our unit, axillary clearance was carried out before referring for radiotherapy.

Neoadjuvant chemotherapy was given to patients with locally advanced disease before surgery. Early stage tumours were treated by surgery followed by adjuvant therapy. Seventy seven of the patients $(62.6 \%)$ had neoadjuvant chemotherapy, a reflection of the level of advanced disease. Unfortunately some patients defaulted when their tumours became smaller and surgery was due. Five of the 77 who had neoadjuvant chemotherapy had complete pathological response (no pathological evidence of cancer in the breast) at mastectomy, showing the effectiveness of the CAF combination therapy used.

Some patients may be suitable for breast reconstruction. It is then preferable to undertake breast reconstruction and mastectomy at the same operative session $^{11,12}$. Several options can be chosen, which range from simple positioning of an expander to the use of musculocutaneous flaps (latissimus dorsi [LD] or Tranverse rectus abdominis myocutaneous [TRAM]), and skin-sparing mastectomy $^{11-13}$. Our plastic surgeons performed LD myocutaneous flaps for 2 patients on request. Breast reconstruction is currently not routinely offered to patients by our unit or other units at the Korle $\mathrm{Bu}$ Teaching Hospital. The provision of more operative time and acquisition of breast reconstruction skills by our breast surgeons would help us perform more reconstruction procedures. It is possible that some our patients refuse mastectomy because they are not offered breast reconstruction.

\section{Defaulting patients}

There was a high rate of default in the patients studied. Twenty patients $(12.7 \%)$ stopped attending as soon as they were informed about the diag- nosis. Fifteen others $(9.5 \%)$ started with neoadjuvant chemotherapy and stopped attending either when there was improvement or when mastectomy was due. For these two groups of patients the fear of or misconceptions about mastectomy were probably the main reasons for defaulting. Six patients $(3.8 \%)$ defaulted and returned after many months with incurable disease. These patients returned with requests for mastectomy for their ulcerated breasts, but were refused surgery because of the advanced nature of the disease and distant metastases.

Education, aimed at dispelling misconceptions about mastectomy and offering breast reconstruction may lead to a reduction in the number of patients defaulting. Surgeons must revise their approach of dealing with breast cancer patients ${ }^{14}$. These patients go through emotions of denial, feeling of injustice, guilt, failure, betrayal and doom ${ }^{14}$. Their cultural beliefs might address their state of vulnerability leading to them turning to traditional healers ${ }^{14}$. Adequate initial counselling is therefore likely to decrease the $12.7 \%$ of patients who stopped attending once the diagnosis was made.

\section{Complications}

The pleura, brain and lungs were the commonest sites of metastases in our patients during followup. Lymphoedema occurred in eleven patients but was serious in only five. No new cases of lymphoedema were detected in patients who had supra/infraclavicular lymph node radiotherapy. The commonest complications following chemotherapy were nausea, vomiting and alopecia. Bone marrow suppression was not marked.

Further studies are needed to determine why patients present late. Patients' fears and misconceptions will have to be addressed in breast awareness education programmes if they are to have an impact. The high rate of default among our patients calls for a more personalized approach for followup, using breast care nurses and counsellors to address patients' concerns, especially the fear of mastectomy.

\section{CONCLUSION}

There has been no improvement in breast cancer presentation at the KBTH over the past 5-10 years, in spite of breast awareness programmes. Patients still present with advanced disease many months after symptoms appear.. The 40-49 decade remains the peak age at presentation. This study has also identified a high rate of default among patients. 
Further research is needed to address these issues and change the trend.

\section{REFERENCES}

1. Badoe EA, and Baako BN. The Breast. In Badoe EA, Archampong EQ and da RochaAfodu (eds) Principles and Practice of Surgery including pathology in the tropics. Accra: Department of Surgery, University of Ghana Medical School. 2000; 449-477.

2. Biritwum RB, Gulaid J, and Amaning A O. Pattern of diseases or conditions leading to hospitalisation at the Korle $\mathrm{Bu}$ Teaching hospital. Ghana Med J 2000; 34:197-205.

3. Archampong EQ. Breast Cancer. Ghana Med $J$ 1977; 16(2): 63 .

4. Asumanu E, Vowotor R, Naaeder SB. Pattern of breast diseases in Ghana. Ghana Med $J$ 2000; 34: 206-209.

5. The Breast. In Greene FL, Page DL, Fleming ID, Fritz AG, Balch CM, Haller DG, Morrow M (eds) AJCC Cancer Staging Manual, $6^{\text {th }}$ Edition. Springer. 2002; 221-245

6. Anim JT. Breast Cancer in Accra. Ghana Med J 1979; 18: 161-167.
7. Hecht J, and Winchester D. Male breast cancer. Am J Clin Pathol 1994, 102: 25-30.

8. Akosa AB, Ampadu FO, Tettey Y. Male Breast Cancers in Ghana. Ghana Med J 1999; 33: $3-8$

9. Kidmas AT, Ugwu BT, Manasseh AN, Iya D, Opaluwa AS. Male breast malignancy in Jos university teaching hospital. West Afr J Med 24(1):36-40.

10. Dixon JM, and Mansel RE. Symptoms, Assessment and guidelines for referral. In Dixon JM (ed). ABC of Breast Diseases. BMJ Publishing Group 1995; 1-6.

11. Veronessi U, Goldhirsch A, Orecchia R, Viale G, Boyle P. Breast Cancer. Lancet 2005; 365 : 1727-1741.

12. Baildam AD. Oncoplastic surgery of the breast. Br J Surg 2002; 89: 532-533.

13. Rainsbury RM. Breast reconstruction - New techniques. In Taylor I, Johnson CD (Eds). Recent advances in Surgery 22. Churchill Livingstone 1999; 17-30.

14. Kaur R. Personal Account - Breast Cancer. Lancet 2005; 365: 1742. 